\title{
Dynamic Spectral Layout of Small Worlds
}

\author{
Ulrik Brandes, Daniel Fleischer, and Thomas Puppe \\ Department of Computer \& Information Science, University of Konstanz, Germany
}

\begin{abstract}
Spectral methods are naturally suited for dynamic graph layout, because moderate changes of a graph yield moderate changes of the layout under weak assumptions. We discuss some general principles for dynamic graph layout and derive a dynamic spectral layout approach for the animation of small-world models.
\end{abstract}

\section{Introduction}

The main problem in dynamic graph layout is the balance of layout quality and mental-map preservation [17. Typically, the problem is addressed by adapting a static layout method such that it produces similar layouts for successive graphs. While these adaptations are typically ad-hoc 8, others 2, 1, are based on the formally derived method [3] of integrating difference metrics [5] into the static method. See 4] for an overview of the dynamic graph drawing problem.

Spectral layout denotes the use of eigenvectors of graph-related matrices such as the adjacency or Laplacian matrix as coordinate vectors. See, e.g., 15] for an introduction. We argue that spectral methods are particularly suited for dynamic graph layout both from a theoretical and practical point of view, because moderate changes in the graph naturally translate into moderate changes of the layout, and updates can be computed efficiently.

This paper is organized as follows. In Sect. 2, we define some basic notation and recall the principles of spectral graph layout. The dynamic graph layout problem is reviewed briefly in Sect. 3, and methods for updates between layouts of consecutive graphs are treated in more detail in Sect. 4. In Sect. 5, our approach for small worlds is introduced, and we conclude with a brief discussion in Sect. 6.

\section{Preliminaries}

For ease of exposition we consider only two-dimensional straight-line representations of simple, undirected graphs $G=(V, E)$ with positive edge weights $\omega: E \rightarrow \mathbb{R}^{+}$, although most techniques and results in this paper easily carry over to other classes of graphs.

In straight-line representations, a two-dimensional layout is determined by a vector $\left(p_{v}\right)_{v \in V}$ of positions $p_{v}=\left(x_{v}, y_{v}\right)$. Most of the time we will reason about one-dimensional layouts $x$ that represent the projection of $p$ onto one component. 
For any graph-related matrix $M(G)$, a spectral layout of $G$ is defined by two eigenvectors $x$ and $y$ of $M(G)$. For simplicity, we will only consider layouts derived from the Laplacian matrix $L(G)$ of $G$, which is defined by elements

$$
\ell_{v, w}= \begin{cases}\sum_{u \in V} \omega(u, v) & , v=w, \\ -\omega(v, w) & , v \neq w,\end{cases}
$$

The rows of $L(G)$ add up to 0 , thus, the vector $\mathbf{1}=(1, \ldots, 1)^{\mathrm{T}}$ is a trivial eigenvector for eigenvalue 0 . Since $L(G)$ is symmetric all eigenvalues are real, and the theorem of Gershgorin 13 yields, that the spectrum is bounded to the interval $[0, g]$, for an upper bound $g \geq 0$. Hence, the spectrum can be written as $0=\lambda_{1} \leq \lambda_{2} \leq \ldots \leq \lambda_{n} \leq g$ with corresponding unit eigenvectors $1 / \sqrt{n}=$ $v_{1}, \ldots, v_{n}$.

Based on the Laplacian, a spectral layout is defined as $p=\left(v_{2}, v_{3}\right)$, where $v_{2}$ and $v_{3}$ are unit eigenvectors to the second and third smallest eigenvalues of the corresponding Laplacian matrix $L(G)$. This has already been used for graph drawing in 1970 by Hall [14.

For sparse graphs of moderate size, a practical method to determine the corresponding eigenvectors is power iteration. For an initial vector $x$ the matrix multiplication $L(G) x /\|L(G) x\|$ is iterated until it converges to a unit eigenvector associated with the largest eigenvalue. Since we are not interested in $v_{n}$, we use matrix $\hat{L}=g \cdot I-L(G)$, which has the same eigenvectors with the order of their eigenvalues $g=g-\lambda_{1} \geq g-\lambda_{2} \geq \ldots \geq g-\lambda_{n}$ reversed. To obtain $v_{2}$ and $v_{3}$, respectively, $x$ is orthogonalized with $v_{1}$ (and in the case of $v_{3}$ also with $v_{2}$ ) after each iteration step, i.e., the mean value $\sum_{i=1}^{n} x_{i} / n$ is subtracted from every element of $x$ to ensure $x \perp \mathbf{1}$. Spectral layouts of larger graphs can be computed efficiently using multiscale methods [16].

\section{Dynamic Layout}

In our setting, a dynamic graph is a sequence $G^{(1)}, \ldots, G^{(r)}$ of graphs with, in general, small edit distance, i.e. $G^{(t)}$ is obtained from $G^{(t-1)}, 1<t \leq r$, by adding, changing, and deleting only a few vertices and edges.

There are two main scenarios for the animation of a dynamic graph, depending on whether the individual graphs are presented to the layout algorithm one at a time, or the entire sequence is known in advance. Layout approaches for the offline scenario (e.g., 7]) are frequently based on a layout of the union of all graphs in the sequence. A variant are $2.5 \mathrm{D}$ representations in which all graphs are shown at once (e.g., 9]). In the online scenario, the typical approach is to consider only the previous layout (e.g., 8]). A variant in which provisions for likely future changes are made is presented in [6].

Since, typically, spectral layouts of similar graphs do not differ much anyway, it is reasonable to ignore the fact that a graph is but one graph in a sequence altogether and compute static layouts for each of them. We rather concentrate on the update step between consecutive layouts. 


\section{Updates}

Assume we are given a sequence of layouts $p_{1}, \ldots, p_{r}$ for a dynamic graph $G^{(1)}, \ldots, G^{(r)}$. The step from $p_{t}$ to $p_{t+1}$ is called logical update, whereas the actual animation of the transition is referred to as the physical update.

While simple, say, linear interpolation of two layouts is most frequently used in graph editors, more sophisticated techniques for morphing are available (see, e.g., [11,12,10]. General morphing strategies do not take into account the method by which origin and target layout are generated.

For dynamic spectral layout, at least two additional strategies are reasonable.

\subsection{Iteration}

If the target layout $x_{t+1}$ is a spectral layout, the iteration for its own computation can and should be initialized with $x_{t}$, that will usually be close to the target layout. The power iteration then produces intermediate layouts which can be used for the physical update. A way to enhance the smoothness of morphing is needed because of the observation, that the first steps of the iteration yield greater movement of the vertices when compared to later steps. Let $\hat{L}=g \cdot I-$ $L\left(G^{(t+1)}\right)$. An iteration step then consists of computing the new layout $\hat{L} x /\|\hat{L} x\|$ from a given layout $x \perp \mathbf{1}$. Let $g=\lambda_{1} \geq \lambda_{2} \geq \ldots \geq \lambda_{n}$ and $\mathbf{1} / \sqrt{n}=v_{1}, v_{2}, \ldots, v_{n}$ be the eigenvalues and unit eigenvectors of $\hat{L}$, respectively. Then if $\lambda_{1}>\lambda_{2}>\lambda_{3}$ (otherwise just proper eigenvectors and eigenvalues would have to be chosen in what follows) and $x=\sum_{i=2}^{n} a_{i} v_{i}, a_{2} \neq 0$ we have

$$
\begin{aligned}
\frac{\hat{L}^{k} x}{\left\|\hat{L}^{k} x\right\|} & \longrightarrow v_{2} \quad \text { and } \\
\left\|v_{2}-\frac{\hat{L}^{k} x}{\left\|\hat{L}^{k} x\right\|}\right\| & =\left\|v_{2}-\frac{\sum_{i=2}^{n} \lambda_{i}^{k} a_{i} v_{i}}{\left\|\hat{L}^{k} x\right\|}\right\| \\
& \leq 1-\frac{\lambda_{2}^{k} a_{2}}{\left\|\hat{L}^{k} x\right\|}+\frac{\sum_{i=3}^{n} \lambda_{i}^{k} a_{i}}{\left\|\hat{L}^{k} x\right\|} \\
& =\mathcal{O}\left(\left(\lambda_{3} / \lambda_{2}\right)^{k}\right) .
\end{aligned}
$$

One way to handle this non-linear decay is to use layouts after appropriately spaced numbers of steps, or to use layouts only if the difference to the last used layout exceeds some threshold $c$ in some metric, e.g., if $\left\|x-x^{\prime}\right\|_{2}>c$. Both ways will enhance the smoothness of morphing by avoiding the drawing of many small movements at the end of the iteration process.

\subsection{Interpolation}

If both origin and target layout $x_{t}$ and $x_{t+1}$ are spectral layouts, intermediate layouts can also be obtained by computing eigenvectors of some intermediate matrices from $L\left(G^{(t)}\right)$ to $L\left(G^{(t+1)}\right)$. We interpolate linearly by

$$
\alpha L\left(G^{(t)}\right)+(1-\alpha) L\left(G^{(t+1)}\right), \quad 1 \geq \alpha \geq 0 .
$$


Layouts are computed for a sequence of breakpoints $1 \geq \alpha_{1}>\alpha_{2}>\ldots>$ $\alpha_{k} \geq 0\left(\alpha_{j+1}-\alpha_{j}\right.$ constant, or proportional to $\sin (\pi j / k)$, depending on what kind of morphing seems to be appropriate, the latter one slowing down at the beginning and end). For every breakpoint $\alpha_{j}$ the iteration is initialized with the layout of $\alpha_{j-1}$, which allows fast convergence and small movements between two succeeding breakpoints. Deletion and insertion of vertices have to be handled in a different manner, since the matrix dimension changes. See Sect. 5 for details.

Figs. 2]and 4 show smooth animations of this method. Theoretical justification for smoothness comes along with a theorem by Rellich [18] applied to the finite dimensional case. Matrix $\alpha L\left(G^{(t)}\right)+(1-\alpha) L\left(G^{(t+1)}\right)$ can be seen as a perturbed self-adjoint operator $L(\varepsilon)=L\left(G^{(t)}\right)+\varepsilon\left(L\left(G^{(t+1)}\right)-L\left(G^{(t)}\right)\right)$ with corresponding eigenvalues $\lambda_{i}(\varepsilon)$ and eigenvectors $v_{i}(\varepsilon)$, that are holomorphic with respect to $\varepsilon$, i.e.

$$
L(\varepsilon) v_{i}(\varepsilon)=\lambda_{i}(\varepsilon) v_{i}(\varepsilon),
$$

where $v_{i}(0)$ are eigenvectors at time $t$ and $v_{i}(1)$ can be permuted by a permutation $\pi$, such that $v_{\pi(i)}(1)$ are (ordered) eigenvectors at time $t+1$. Note that two eigenvectors may only have to be exchanged if its corresponding eigenvalues intersect during the time from $t$ to $t+1$. And even then the power iteration exchanges these eigenvectors sufficiently smooth for pleasing animations, because the corresponding eigenvalues remain within the same range for some time due to smooth functions $\lambda_{i}(\varepsilon)$. Consider $\lambda_{2}$ and $v_{2}$ of the following small-world example with $n=100$ vertices and $k=7$, where starting from a circle each vertex is connected to its $2 k$ nearest neighbors. Both $\lambda_{2}(\varepsilon)$ and $v_{2}(\varepsilon)$ can locally be written as power series

$$
\begin{aligned}
& \lambda_{2}(\varepsilon)=\mu_{0}+\varepsilon \mu_{1}+\varepsilon^{2} \mu_{2}+\ldots, \\
& v_{2}(\varepsilon)=w_{0}+\varepsilon w_{1}+\varepsilon^{2} w_{2}+\ldots .
\end{aligned}
$$

We show that $\| w_{i}|| \leq 1$ and $\left|\mu_{i}\right| \leq 2 / \sqrt{n}$ for $i>0$, hence $\lambda_{2}(\varepsilon)$ and $v_{2}(\varepsilon)$ will be smooth functions, say, within $[0,1 / 2]$ (and by the same construction within the remaining interval, too). We write $L(\varepsilon)=L+\varepsilon P$, where $P$ is the insertion of an edge between two non-adjacent vertices (with indices 1 and $r$ ), and denote by $I$ the identity matrix. From (11) and (2) we get

$$
\begin{aligned}
L w_{0} & =\mu_{0} w_{0}, \\
\left(L-\mu_{0} I\right) w_{j} & =\sum_{i=0}^{j-1} \mu_{j-i} w_{i}-P w_{j-1},(j>0),
\end{aligned}
$$

where we can recursively choose $w_{j},(j>0)$ such that $w_{j} \perp w_{0}$. Since the right hand sides of (3) need to be orthogonal to $w_{0}$ for $j>0$ this yields

$$
\mu_{j}=\left\langle P w_{j-1}, w_{0}\right\rangle,(j>0) .
$$

Now we can recursively compute upper bounds for $\left|\mu_{j}\right|$ and $\left\|w_{j}\right\|$. Note that $\lambda_{2}$ has multiplicity 2 , and the right hand sides of (3) are also orthogonal to $v_{1}$, such 
that $1 /\left(\lambda_{4}-\lambda_{2}\right)$ is the least upper bound of the inverse mapping of $L-\mu_{0} I$ applied to the right hand side.

$$
\begin{aligned}
\left|\mu_{1}\right| & =\left(w_{0,1}-w_{0, r}\right)^{2} \leq 2 / n \leq 2 / \sqrt{n} \\
|| w_{1} \| & \leq \frac{2\left|w_{0,1}-w_{0, r}\right|}{\lambda_{4}-\lambda_{2}} \leq \frac{2 \sqrt{2 / n}}{1.568}=: c \leq 1 / 4.35, \\
\left|\mu_{j}\right| & \leq 2 \kappa_{j-1} c^{j-1} / \sqrt{n} \leq 2 / \sqrt{n} \\
|| w_{j} \| & \leq \kappa_{j} c^{j} \leq 1
\end{aligned}
$$

where $\kappa_{j}$ is defined by $\kappa_{0}=1$ and $\kappa_{j}=\sum_{i=0}^{j-1} \kappa_{i} \kappa_{j-i-1}$ for $j>0$.

Lemma 1. $\kappa_{j} \leq 4.35^{j}$.

Proof. We show $\kappa_{j} \leq 4.35^{j} /(j+1)^{2}$, which holds for all $j<144$ (by evaluating). For $j \geq 144$

$$
\begin{aligned}
\kappa_{j} & \leq \sum_{i=0}^{j-1} \frac{4.35^{i}}{(i+1)^{2}} \cdot \frac{4.35^{j-i-1}}{(j-i)^{2}} \leq 2 \cdot 4.35^{j-1} \sum_{i=0}^{\lfloor j / 2\rfloor} \frac{1}{(i+1)^{2}} \cdot \frac{1}{(j-i)^{2}} \\
& \leq 2 \cdot 4.35^{j-1}\left(\frac{1}{(j-9)^{2}} \sum_{i=1}^{10} \frac{1}{i^{2}}+\frac{4}{j^{2}}\left(\zeta(2)-\sum_{i=1}^{10} \frac{1}{i^{2}}\right)\right) \leq \frac{4.35^{j-1}}{(j+1)^{2}} \cdot 4.348
\end{aligned}
$$

Note that $\left\|w_{j}\right\| \leq 1$ could also be shown for much weaker assumptions than $c \leq 1 / 4.35$, which was sufficient for our example. Lemma 1 is only very close to optimal, the least upper bound of $1 /\left(\lambda_{4}-\lambda_{2}\right)$ is in general not achieved, and $\left|\mu_{j}\right|=\left|\left\langle P w_{j-1}, w_{0}\right\rangle\right|$ can in general be better bounded than by $\| w_{j-1}|| \sqrt{4 / n}$.

\section{Application to Small Worlds}

Spectral layout methods are naturally suited for smooth dynamic layout, because the influence of vertices and edges that are subject to change can be increased or decreased gradually. Moreover, each can be determined by iterative computations that benefit from good initialization, so that moderate changes leads to moderate and efficient updates.

Watts and Strogatz [19] introduced a random graph model that captures some often-observed features of empirical graphs simultaneously: sparseness, local clustering, and small average distances. This is achieved by starting from a cycle and connecting each node with its $2 k$ nearest neighbors for some small, fixed $k$. The resulting graph is sparse and has a high clustering coefficient (average density of vertex neighborhoods), but also high (linear) average distance.

The average distance drops quickly when only a few random edges are rewired randomly. If each edge is rewired independently with some probability $p$, there is a large interval of $p$ in which the average distance is already logarithmic while the clustering coefficient is still reasonably high. 


\subsection{Dynamic Laplacian Layout}

Interestingly, spectral layouts highlight the construction underlying the above model and thus point to the artificiality of generated graphs. This is due to the
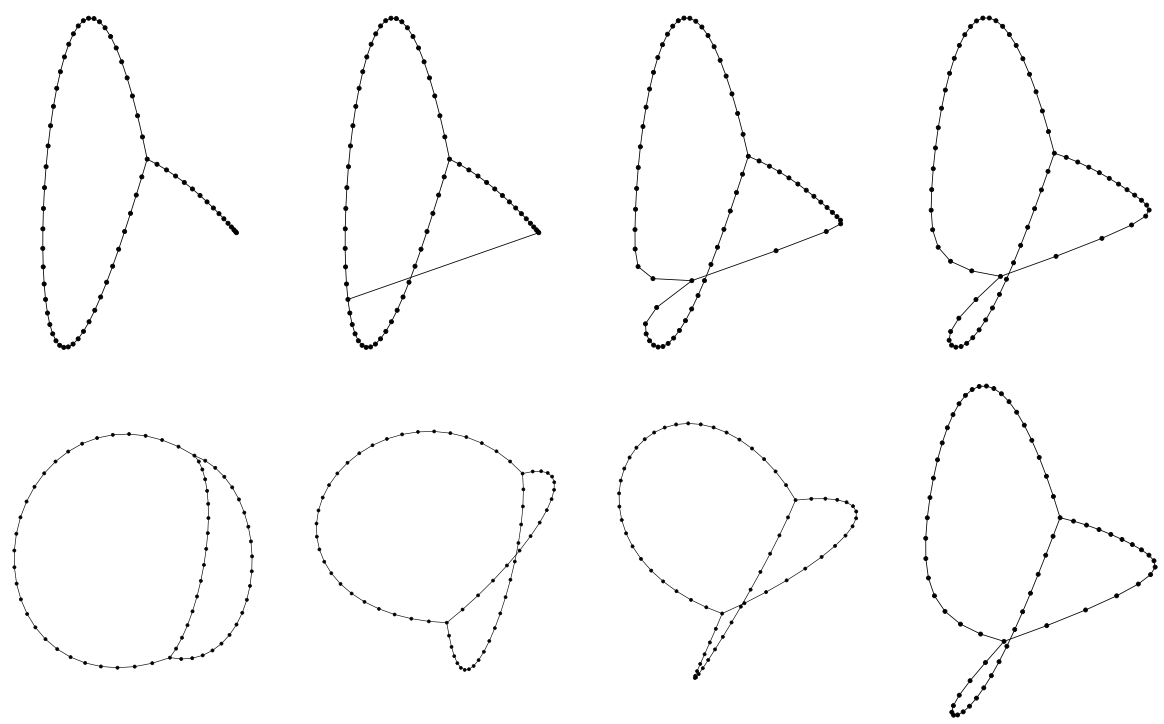

Fig. 1. Update by iteration (read top left to top right to bottom right to bottom left). Note the spread of change along the graph structure.
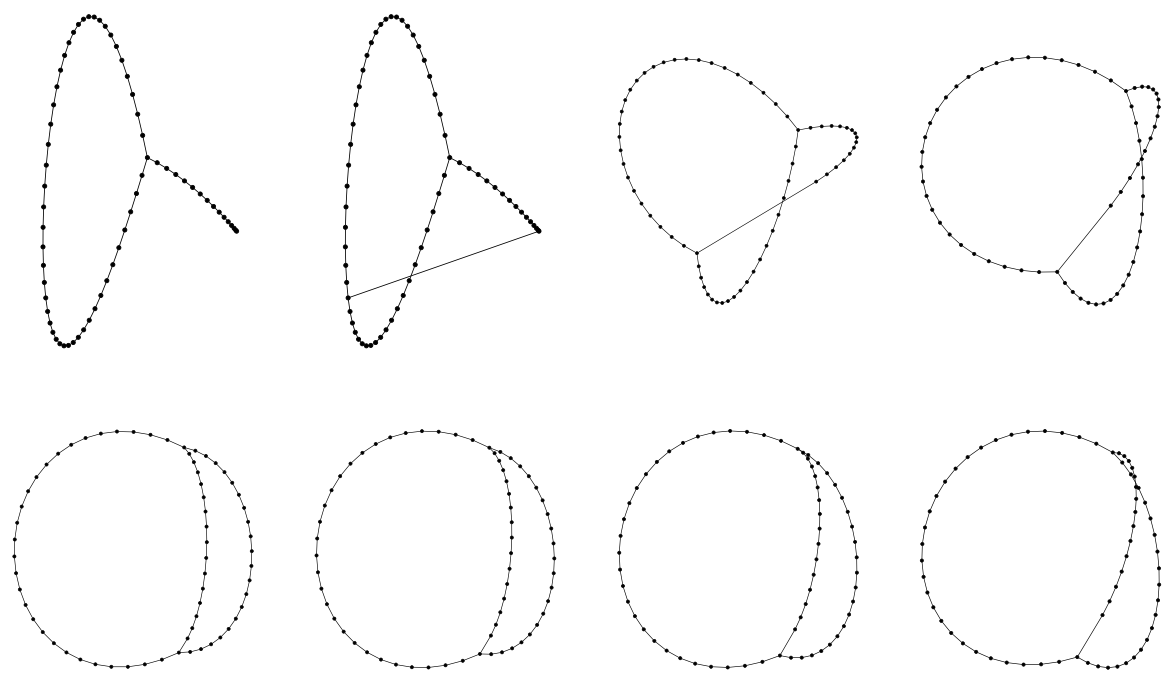

Fig. 2. Update by interpolation. Layout anomalies are restricted to modified part of graph. 
fact that spectral layouts of regular structures display their symmetry very well, and are only moderately disturbed by small perturbations in the graph (mirroring the argument for their use in dynamic layout). The initial ring structure of the small world in Fig. 5 is therefore still apparent, even though a significant number of chords have been introduced by random rewiring. In fact, the layout conveys very well which parts of the ring have been brought together by short-cut edges.

Figs. 1 and 2 point out differences between the two approaches using intermediate layouts obtained from the power iteration and from matrix interpolation.
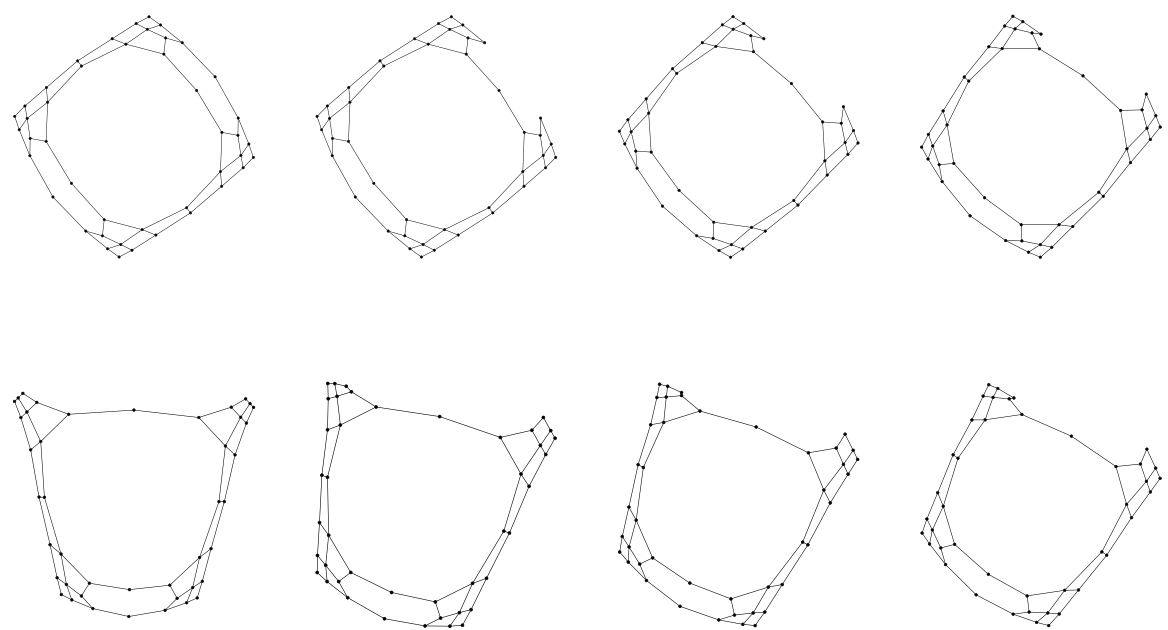

Fig. 3. Update by simple linear interpolation. Intermediate layouts are less symmetric.
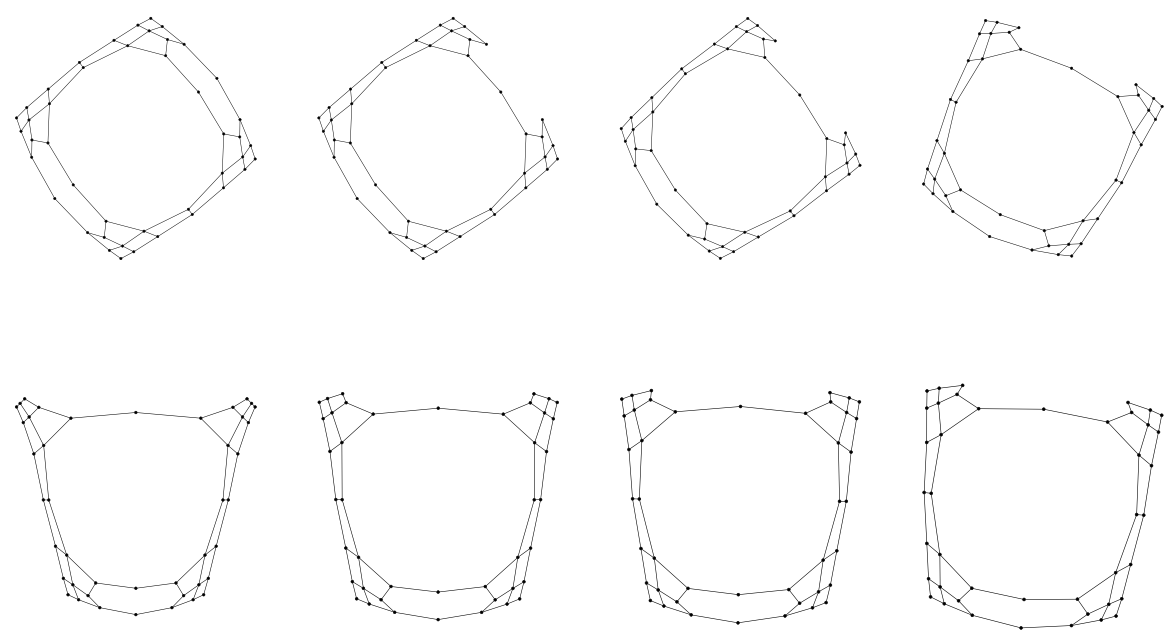

Fig. 4. Interpolation updates maintain symmetry 

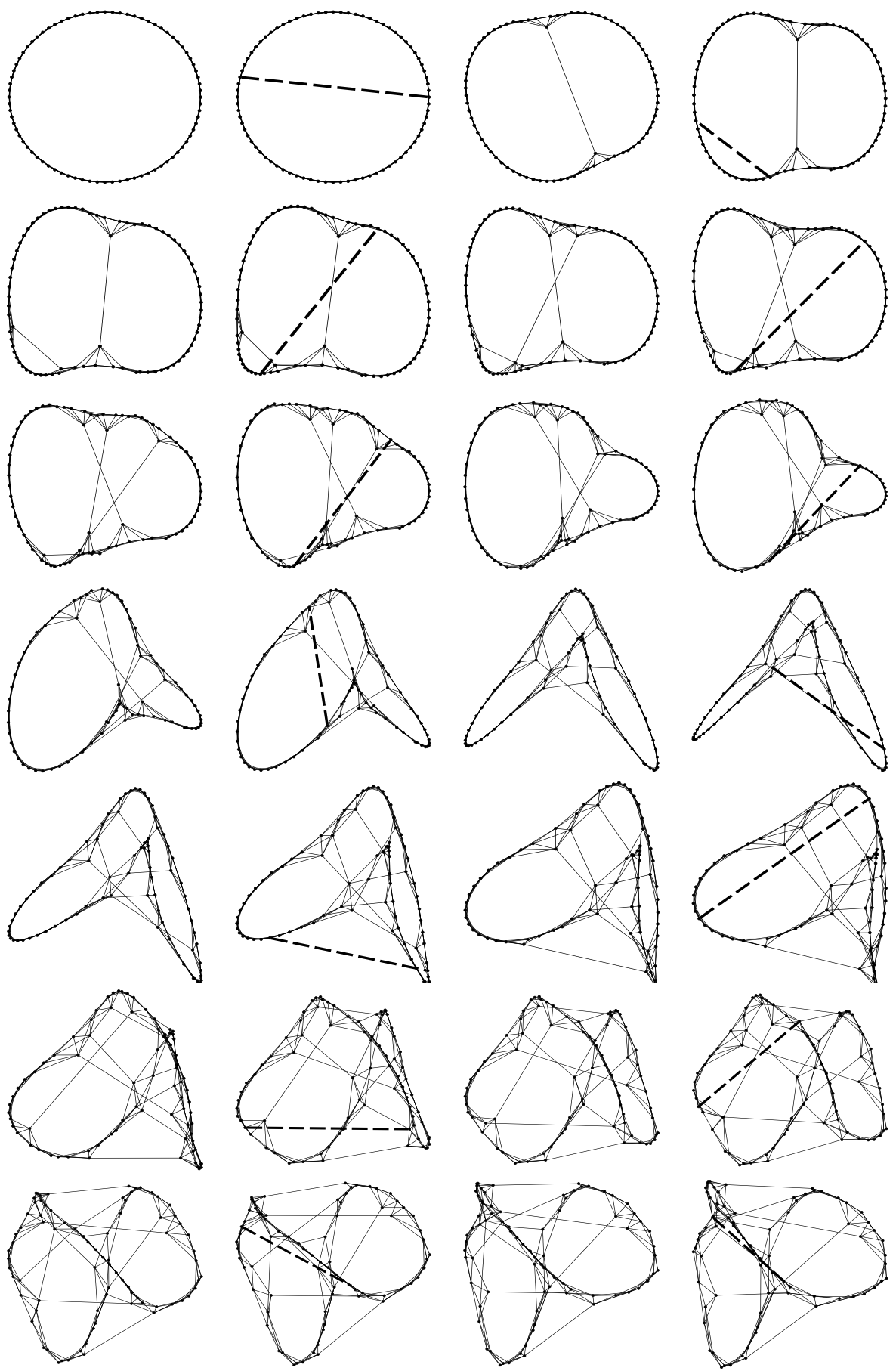

Fig. 5. Evolution of a small world (read top to bottom, left to right) 
It can be seen that the power iteration first acts locally around the changes. This stems from the fact that in the first multiplication only the neighborhood of the change, i.e., the two incident vertices of an edge with changed weight or the neighbors of a deleted or inserted vertex, is affected. The next step also affects vertices at distance 2, and so on. Hence, the change spreads like a wavefront. The matrix interpolation approach acts globally at every step. Interpolating the Laplacian matrices corresponds to gradually changing edge weights. The animation therefore is much more smooth.

Figs. 3 and 4 show differences between simple linear interpolation of the positions and matrix interpolation. In Fig. 3 can be seen, that the symmetry of the graph to its vertical axis is not preserved during the animation, whereas in Fig. 4 each intermediate layout preserves this symmetry.

Fig. 5 finally shows some snapshots of a small world evolving from a torus. The layouts were obtained by using matrix interpolation (one intermediate step per change shown). Note that deletion and insertion of vertices requires some extra efforts, in particular, if the deletion of a vertex disconnects the graph.

\subsection{Deletion and Insertion of Vertices}

Consider deletion of a single vertex $v$, that does not disconnect the graph. Matrix $L\left(G^{(t+1)}\right)$ is then expanded by one row and column of zeros corresponding to vertex $v$, such that $L\left(G^{(t)}\right)$ and $L\left(G^{(t+1)}\right)$ have the same dimension. This derived matrix has a double eigenvalue 0 . A new corresponding eigenvector is, e.g., $(0, \ldots, 0,1,0, \ldots, 0)^{\mathrm{T}}$, where the 1 is at position corresponding to $v$. This eigenvector will cause vertex $v$ to drift away during power iteration, and thus all other vertices stick together. This can be prevented by defining $\ell_{v, v}=g$ in matrix $L\left(G^{(t+1)}\right)$, leading to a movement of $v$ towards 0 . But in practice, the following method proved to be successful. After every matrix multiplication reset the position of $v$ to the barycenter of its neighbors. This either prevents a drifting away or an absorbing to 0 , which would otherwise be hard to manage. Apart from using matrix $\alpha L\left(G^{(t)}\right)+(1-\alpha) L\left(G^{(t+1)}\right)$ for the power iteration, orthogonalization and normalization also have to be adapted. For time $t+1$ we only need $x_{t+1} \perp(1, \ldots, 1,0,1, \ldots, 1)$, instead of $x_{t+1} \perp \mathbf{1}$, and only the restriction to the elements not corresponding to $v$ have to be normalized. Both can be done by linear interpolation of these operations.

Insertion of a vertex $v$ is treated analogously. Expand matrix $L\left(G^{(t)}\right)$ by one row and column of zeros as above. Orthogonalization and normalization again have to be adapted.

\subsection{Disconnected Graphs}

The deletion of a cut vertex (or a bridge) disconnects the graph $G^{(t+1)}$ into $k \geq 2$ components $G_{1}, \ldots, G_{k}$. Each component is drawn separately by spectral methods and afterwards these layouts are merged to a layout for $G^{(t+1)}$. Basically, there are three parameters for each component, that have to be determined after a layout $x_{j}$ for each $G_{j}$ was computed. The first one determines the size of 
each component, i.e., find a constant $s_{j}$, that scales $x_{j}$ to $s_{j} x_{j}$. The second one determines where the barycenter of each component is set to. The rotation angle of each component could also be considered, but we concentrate on the first two parameters only.

The removal of a cut vertex (or a bridge) yields a matrix $L\left(G^{(t+1)}\right)$, that, after rearranging, consists of $k$ blocks $L_{1}, \ldots, L_{k}$, which are Laplacian matrices of lower dimensions

$$
L\left(G^{(t+1)}\right)=\left(\begin{array}{cccc}
L_{1} & & & 0 \\
& L_{2} & & \\
& & \ddots & \\
& & & L_{k}
\end{array}\right) .
$$

Each of the components is now drawn separately, simply by the common power iteration of the whole matrix $L\left(G^{(t+1)}\right)$, where only normalization and orthogonalization have to be modified appropriately. The barycenter $c_{j}$ of each component thus is 0 , which we now reset to a new position. For notational purposes identify the 2-dimensional plane with complex numbers. Let the current barycenters $c_{j}$ be sorted increasingly by their angle to the positive real axis and reset them to

$$
c_{j}:=\frac{\eta}{2 \sqrt{2}} \exp \left(\frac{2 \pi i}{\eta}\left(-\frac{\eta_{j}}{2}+\sum_{\ell=1}^{j} \eta_{\ell}\right)\right), \quad \eta_{j}=\sqrt{\frac{\left|G_{j}\right|}{\left|G^{(t+1)}\right|}}, \quad \eta=\sum_{j=1}^{k} \eta_{j} .
$$

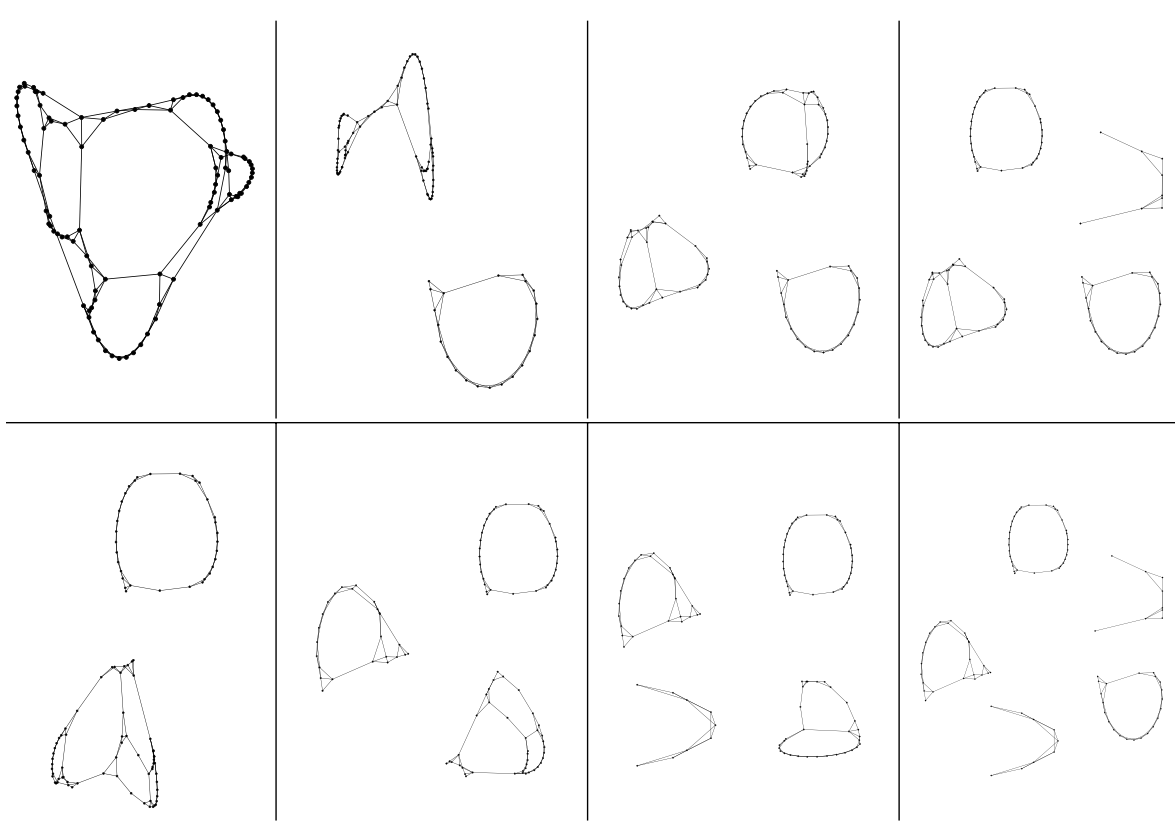

Fig. 6. Drawing connected components (top: left to right, bottom: right to left) 
Together with a normalization $s_{j}=\eta_{j}$, this has the effect, that the components are distributed on a circle with radius $\eta /(2 \sqrt{2})$, each on an area proportional to the size of the component, and none of them overlap. Depending on the shape of the components the radius can also be decreased. Note that the normalization is also well suited, because $\sum_{j=1}^{k} \eta_{j}^{2}=1$ - analogous to $\|x\|=1$.

Altogether, when removing a cut vertex, new barycenters are computed, power iteration with modified orthogonalization/normalization is applied, and meanwhile each component moves to its new barycenter linearly to the chosen breakpoints.

Further splitting and merging of connected components are handled analogously, see Fig. 6] for an example.

\section{Discussion}

We have proposed a dynamization scheme for spectral layout and applied it to changing small-world graphs. While there is no need to make special provisions for logical updates, it turns out that matrix interpolation is the method of choice for the physical update. Despite its simplicity, the scheme achieves both static layout quality and mental-map preservation, because it utilizes stability inherent in spectral layout methods.

Much of the dynamization scheme directly applies to force-directed methods as well, and is in fact driven by common practices [8].

For both spectral and force-directed layout update computations are rather efficient, since the preceding layouts are usually very good initializations for iterative methods. For large graphs, it will be interesting to generalize the approach to multilevel methods, possibly by maintaining (at least part of) the coarsening hierarchy and reusing level layouts for initialization.

In general, spectral layouts are not suitable for graphs with low connectivity, even in the static case. However, our dynamic approach is likely to work with any improved methods for static spectral layout as well.

\section{References}

1. U. Brandes, M. Eiglsperger, M. Kaufmann, and D. Wagner. Sktech-driven orthogonal graph drawing. In Proc. GD 2002, LNCS 2528, pages 1-11. Springer, 2002.

2. U. Brandes, V. Kääb, A. Löh, D. Wagner, and T. Willhalm. Dynamic WWW structures in 3D. Journal of Graph Algorithms and Applications, 4(3):103-114, 2000 .

3. U. Brandes and D. Wagner. A Bayesian paradigm for dynamic graph layout. In Proc. GD 1997, LNCS 1353, pages 236-247. Springer, 1997.

4. J. Branke. Dynamic graph drawing. In M. Kaufmann and D. Wagner, editors, Drawing Graphs, LNCS 2025, pages 228-246. Springer, 2001.

5. S. Bridgeman and R. Tamassia. Difference metrics for interactive orthogonal graph drawing algorithms. Journal of Graph Algorithms and Applications, 4(3):47-74, 2000 . 
6. C. Demestrescu, G. Di Battista, I. Finocchi, G. Liotta, M. Patrignani, and M. Pizzonia. Infinite trees and the future. In Proc. GD 1999, LNCS 1731, pages 379-391. Springer, 1999.

7. S. Diehl, C. Görg, and A. Kerren. Preserving the mental map using forsighted layout. In Proc. VisSym 2001. Springer, 2001.

8. P. Eades, R. F. Cohen, and M. Huang. Online animated graph drawing for web navigation. In Proc. GD 1997, LNCS 1353, pages 330-335. Springer, 1997.

9. C. Erten, P. J. Harding, S. G. Kobourov, K. Wampler, and G. Yee. GraphAEL: Graph animations with evolving layouts. In Proc. GD 2003, LNCS 2912, pages 98-110. Springer, 2003.

10. C. Erten, S. G. Kobourov, and C. Pitta. Intersection-free morphing of planar graphs. In Proc. GD 2003, LNCS 2912, pages 320-331. Springer, 2003.

11. C. Friedrich and P. Eades. Graph drawing in motion. Journal of Graph Algorithms and Applications, 6(3):353-370, 2002.

12. C. Friedrich and M. E. Houle. Graph drawing in motion II. In Proc. GD 2001, LNCS 2265, pages 220-231. Springer, 2001.

13. G. H. Golub and C. F. van Loan. Matrix Computations. John Hopkins University Press, 1983.

14. K. M. Hall. An $r$-dimensional quadratic placement algorithm. Management Science, 17(3):219-229, 1970.

15. Y. Koren. Drawing graphs by eigenvectors: Theory and practice. Computers and Mathematics with Applications, 2005. To appear.

16. Y. Koren, L. Carmel, and D. Harel. Drawing huge graphs by algebraic multigrid optimization. Multiscale Modeling and Simulation, 1(4):645-673, 2003.

17. K. Misue, P. Eades, W. Lai, and K. Sugiyama. Layout adjustment and the mental map. Journal of Visual Languages and Computing, 6(2):183-210, 1995.

18. F. Rellich. Perturbation Theory of Eigenvalue Problems. Gordon and Breach Science Publishers, 1969.

19. D. J. Watts and S. H. Strogatz. Collective dynamics of "small-world" networks. Nature, 393:440-442, 1998. 